\title{
Dermatofibrosarcoma Protuberans: A Rare Malignancy
}

\author{
Ümmügül ÜYETÜRK, ${ }^{1}$ Tuba TASLAMACIOĞLU DUMAN, ${ }^{2}$ Selma ERDOĞAN DÜZCÜ, ${ }^{3}$ \\ Nadire KÜÇÜKÖZTAŞ, ${ }^{1}$ Fahri YILMAZ
}

'Department of Medical Oncology, Abant Izzet Baysal University, Faculty of Medicine, Bolu, Turkey

${ }^{2}$ Department of Internal Medicine, Abant Izzet Baysal University, Faculty of Medicine, Bolu, Turkey

${ }^{3}$ Department of Pathology, Abant Izzet Baysal University, Faculty of Medicine, Bolu, Turkey

\begin{abstract}
OBJECTIVE
Dermatofibrosarcoma protuberans (DFSP) is a fibrohistiocytic tumor characterized by protuberant growth pattern of skin and subcutaneous tissue. The annual incidence is 4.2 per million. It is often seen between the ages of 20-50 years. This study aimed to evaluate the characteristics of patients with DFSP.

METHODS

Patients who visited our clinic were retrospectively evaluated.

\section{RESULTS}

All patients were females and the median age was 42 (29-60) years. The evaluated tumor sites were observed in the body/trunk in one patient, at the hip in two, and at the proximal lower extremity in two. Surgical excision was done in all of them as initial treatment. Re-excision was needed in three patients. One of the patients underwent punch biopsy for diagnosis and surgical removal of the tumor with a negative surgical margin. One of the patients underwent radiotherapy. The median follow-up time was 10 (6-26) months. During the median follow-up, there was no local recurrence or metastasis.
\end{abstract}

\section{CONCLUSION}

As initial treatment, it is important to surgically remove tumors that had no distant metastasis. Positive or near surgical margins should be resected again. If a negative surgical margin cannot be achieved, radiotherapy should be used as the treatment. Imatinib mesylate is used for treatment in unresectable, recurrent, and/or metastatic tumors.

Keywords: Dermatofibrosarcoma protuberans; imatinib; radiotherapy; surgery.

Copyright ( ) 2017, Turkish Society for Radiation Oncology

\section{Introduction}

Dermatofibrosarcoma protuberans (DFSP) is a rare soft tissue sarcoma with a low to moderate grade.[13] It constitutes $1 \%-6 \%$ of all soft tissue sarcomas. [4] The annual incidence is 4.2 per million. It is 1.14 times more common in females than males.[5] Although it can be seen at any age, the incidence is often increased between 20 and 50 years.[6]
DFSP, a fibrohistiocytic tumor characterized by skin and subcutaneous protuberant growth pattern, has painless, slow-growing, dark red or blue plaques. Over time, tumor enlargement and protuberant or ulcerative appearance occurs. Protuberant nodules can infiltrate subcutaneous tissue, fascia, muscles, and even bone.[7]

DFSP is characterized by translocation between chromosomes 17 and 22 [t (17;22) (q22; q13)], indicating platelet-derived growth factor receptor beta (PDG- 


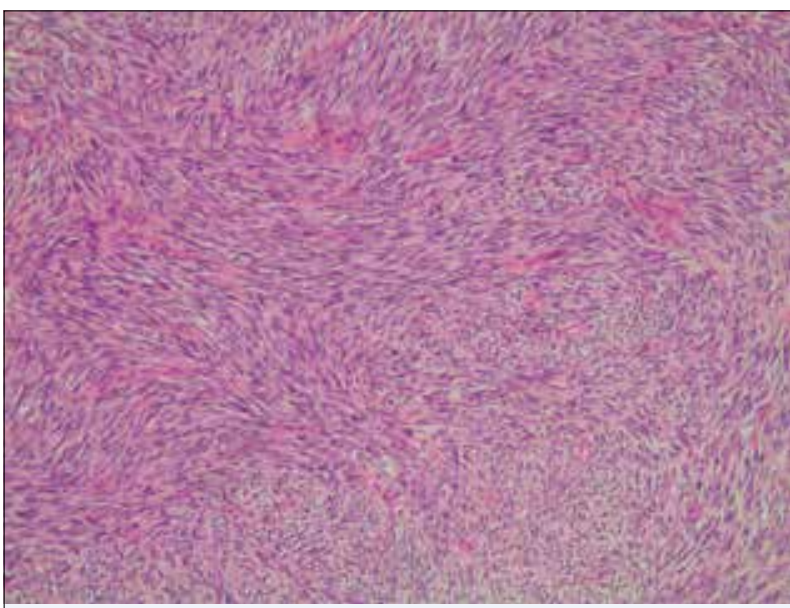

Fig. 1. A storiform pattern of spindle cells in the dermis $(\mathrm{H} \& \mathrm{E}, \times 100)$.

FRB) overexpression. Patients with this translocation can be treated with imatinib mesylate.[8]

It is important to surgically remove tumors with no metastasis during the initial treatment. If the tumor is not completely removed, the recurrence rate is high. Positive or near surgical margins should be resected again. $[6,9,10]$ If a negative surgical margin cannot be achieved, radiotherapy should be preferred or imatinib mesylate should be used as medical treatment.[11] If recurrences occur, re-excision or radiotherapy should be performed. Imatinib mesylate is also used in metastatic tumors in which resection is not possible or may have unacceptable functional and cosmetic results and radiotherapy cannot be performed.[12-14]

This study aimed to review the literature for DFSP cases.

\section{Materials and Methods}

Between January 1, 2012 and December 31, 2016, patients diagnosed with DFSP who applied to the medical oncology clinic of Abant Izzet Baysal University Medical Faculty were retrospectively evaluated. Age, sex, tumor location, pathologic features, treatment, follow-up time, recurrence or metastasis, and current status were recorded.

\section{Results}

During this time we had five patients with DFSP whose general features were defined as given below.

Case 1: A 60-year-old female patient with complaint of blue-violet painless swelling on the right side of her

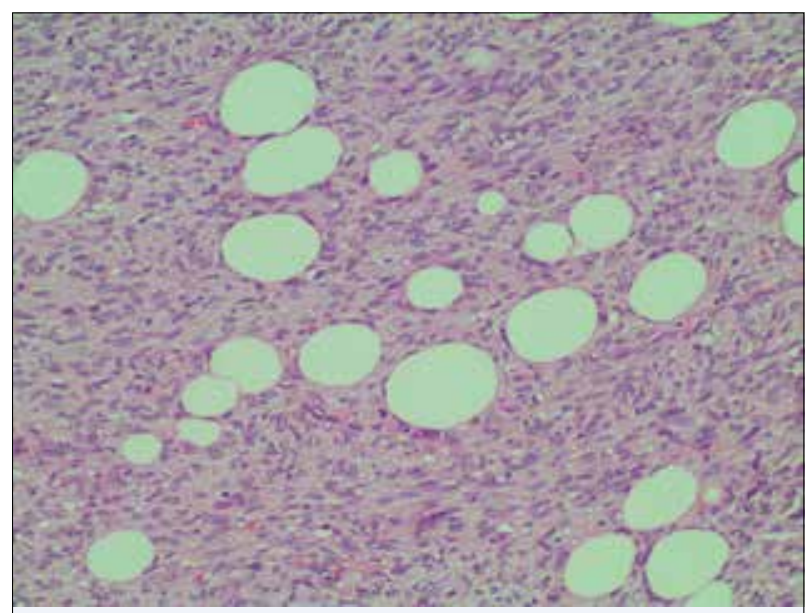

Fig. 2. Dermatofibrosarcoma protuberans invading into the underlying subcutaneous fat $(H \& E, \times 200)$.

waist for about 6 months presented to the clinic. Her past history revealed that 28 months ago, she had a surgical intervention for the complaint of swelling at the same site, which was pathologically diagnosed as fibroma. There was no property in her family history. The patient's excised tumor measured $5 \times 2.5 \times 5 \mathrm{~cm}$ and was microscopically infiltrated with subcutaneous fat tissue starting just below the multilamellar epithelium and high-cell tumor with a cellularity showing storiform pattern was observed (Fig. 1 and Fig. 2).Tumor cells were pale, eosinophilic, with clear cytoplasm and hyperchromatic nuclei. Common mitoses were observed in tumor cells. The tumor was adjacent to the basal and lateral surgical margins. Immunohistochemical (IHC) staining showed that tumor cells were CD34 positive (Fig. 3), factor XIIIa negative (Fig. 4), CD68 negative, and S100 negative. Ki-67 proliferation index was evalu-

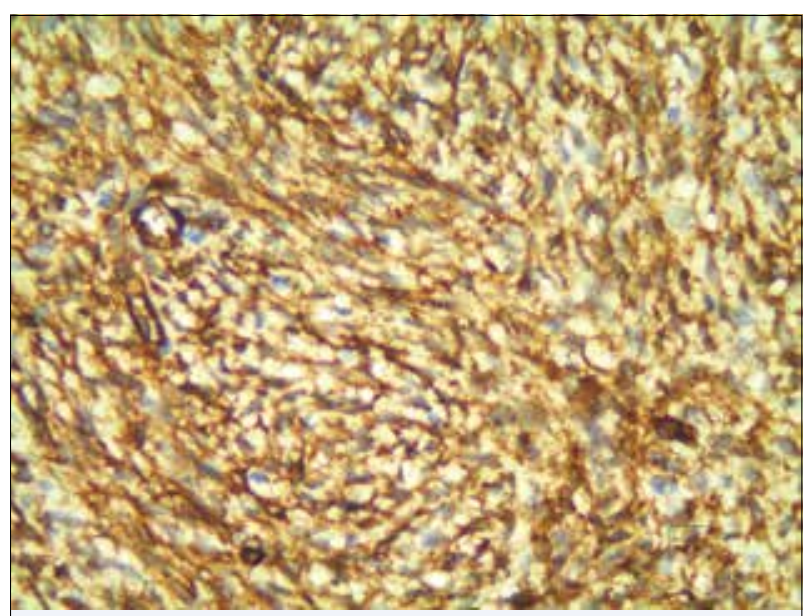

Fig. 3. CD 34 positive staining of the lesion $(\times 400)$. 
Table 1 General patient characteristics

\begin{tabular}{|c|c|c|c|c|c|}
\hline & Case 1 (HA) & Case 2 (EK) & Case 3 (ŞA) & Case 4 (FA) & Case 5 (EY) \\
\hline Age (year) & 60 & 60 & 42 & 44 & 29 \\
\hline Sex & Female & Female & Female & Female & Female \\
\hline Site & Trunk & $\begin{array}{c}\text { Proximal } \\
\text { Lower Extremity }\end{array}$ & Hip & $\begin{array}{c}\text { Proximal Lower } \\
\text { Extremity }\end{array}$ & Hip \\
\hline Surgery & Yes & Yes & Yes & Yes & Yes \\
\hline Surgical margin & $\begin{array}{c}\text { Negative } \\
\text { (Re-excision) }\end{array}$ & Positive & $\begin{array}{c}\text { Negative } \\
\text { (Re-excision) }\end{array}$ & $\begin{array}{c}\text { Negative } \\
\text { (Re- excision) }\end{array}$ & Negative \\
\hline Tumor diameter $(\mathrm{cm})$ & $5 \times 2.5 \times 5$ & $2.1 \times 1.5 \times 1.3$ & $2 \times 1 \times 1.2$ & $1.5 \times 1 \times 1$ & $0.7 \times 0.5 \times 0.5$ \\
\hline CD 34 & Positive & Positive & Positive & Positive & Positive \\
\hline Factor XIIIa & Negative & Negative & No stain & Negative & Negative \\
\hline Ki-67(\%) & 60 & 8 & 2 & Not evaluated & Not evaluated \\
\hline Radiotherapy & No & Yes & No & No & No \\
\hline Systemic treatment & No & No & No & No & No \\
\hline Time to metastasis (month) & 0 & 0 & 0 & 0 & 0 \\
\hline Follow-up time(month) & 26 & 10 & 9 & 6 & 22 \\
\hline Current status & Alive & Alive & Alive & Alive & Alive \\
\hline
\end{tabular}

ated as $60 \%$. The patient had no metastasis and was followed up after a negative surgical margin was achieved.

Case 2: A 60-year-old woman presented with painless swelling on the side of the right thigh for about 7 months. The patient's history and family history were unremarkable. The excised tumor of the patient measured $2.1 \times 1.5 \times 1.3 \mathrm{~cm}$. Microscopic examination revealed a tumor showing infiltration in the fatty tissue with ulcerations in the multilamellar squamous epithelium. Tumor cells were arranged in a storiform pattern that crossed each other. Cells were spindle shaped with epithelioid character with hyperchromatic nucleus and narrow cytoplasm. Tumor cells were positive for CD34 and vimentin, negative for factor XIIIa, S100, HMB45,

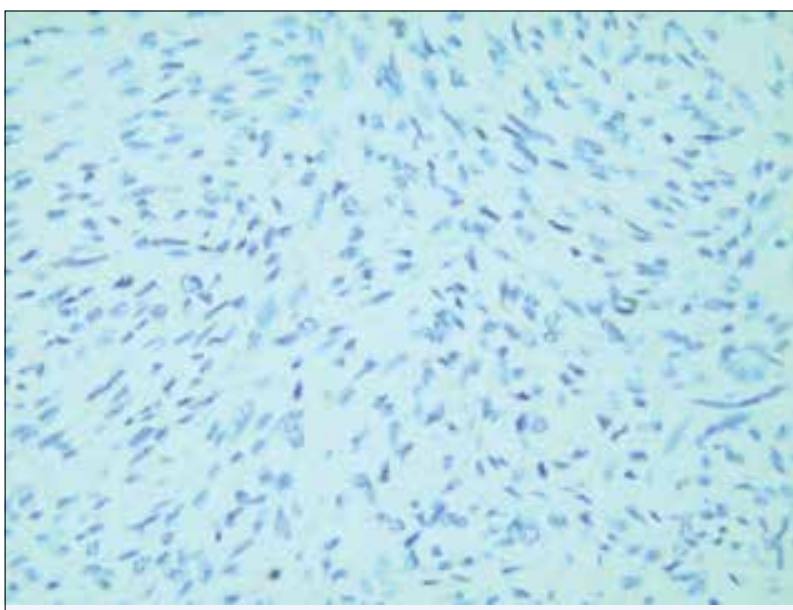

Fig. 4. Factor XIIIa negative staining of the lesion $(\times 400)$. and desmin in IHC staining. Ki-67 proliferation index was evaluated as $8 \%$. Radiotherapy was performed because it was thought that surgery could not be achieved with re-excision for the patient with positive surgical margin. Patient had no metastasis and was followed up.

Case 3: A 42-year-old woman who had a swelling on her right hip for about 20 years was suffering from the swelling's changing color and enlargements for the past year. She has no other feature, except for the solitary nodule in the thyroid. Her parents had died due to lung cancer. Her brother had thyroid cancer. The excised tumor measured $2 \times 1 \times 1.2 \mathrm{~cm}$ and on microscopic examination revealed infiltration in the fatty tissue that formed ulceration in the multilamellar epithelium. Tumor cells were arranged in a storiform pattern that crossed each other. Cells were spindle shaped with epithelioid character with hyperchromatic nucleus and narrow cytoplasm. Tumor cells were CD34 positive in the IHC stain. Ki-67 proliferation index was evaluated as $2 \%$. Re-excision was performed in the patient with a positive surgical margin. Patient had no metastasis and was followed up after successful surgery with negative surgical margin.

Case 4: A 44-year-old woman with a history of swelling in the right and left thighs for about 6 years was suffering from the swelling's changing color and enlargements for the last 2 months, especially in the left thigh. The patient had a history of epilepsy. Her family history was unremarkable. A well-limited fibrohis- 
tiocytic benign tumor dermatofibroma in the dermis excised from the right thigh and a $1.5 \times 1 \times 1-\mathrm{cm}$ tumor in the left thigh was evaluated as DFSP with infiltrative appearance of the dermis, a storiform pattern, and spindle cells. IHC staining showed CD34 positive and factor XIIIa negative. Re-excision was performed with a negative surgical margin, and after surgery, the patient had no metastasis and was followed up.

Case 5: A 29-year-old woman who had a swelling on her right hip for about 3 years was suffering from the swelling's changing color for the last 3 months. There was a history of Familial Mediterranean Fever. Her family history was unremarkable. Punch biopsy was done. Spindle cellular tumor was found in the storiform pattern, extending from the submucosal fatty tissue, starting from the multilamellar flat epithelium. The IHC stain was positive for CD34 and vimentin and negative for factor XIIIa and S100. Surgical margin negativity was ensured and tumor was removed with the largest diameter was $0.7 \times 0.5 \times 0.5 \mathrm{~cm}$. The patient had no metastasis and was followed up (Table 1).

\section{Discussion}

DFSP is a low to intermediate grade fibrohistiocytic malignant tumor growing as a nodular cutaneous mass. [4] DFSP is a rare soft tissue sarcoma that is frequently seen between 20 and 50 years of age and occurs more frequently in females than in males and in the trunk than in extremities. [1,5,6] Our cases were in the age range consistent with the literature. Unlike the literature, trunk placement was present in only one patient, and all cases were female.

This tumor may be misdiagnosed as a result of inappropriate tissue sampling or superficial biopsy. Deep subcutaneous layer punch or incisional biopsy is recommended for adequate tissue sampling and complete pathologic evaluation.[15] In our cases, tumors were surgically removed without biopsy in four cases. But surgical margin negativity could not be achieved, except for one patient. The others needed re-excision. One patient's surgery was done after punch biopsy and she did not need re-excision. It was thought that diagnosis by punch or incisional biopsy before surgery was important in terms of providing surgical margin negativity. In fact, dermatofibroma differs from DFSP at times. In such cases CD34, factor XIIIa, nestin, apolipoprotein $\mathrm{D}$, cathepsin staining are useful as IHC. CD34 positivity and factor XIIIa negativity was seen in almost all cases with DFSP diagnosis. Ki-67 is used as a proliferation marker.[15,16] In our cases, histological pa- rameters such as the absence of lesion area between epidermis and tumor, diffuse infiltration into subcutaneous fatty tissue, and irregularity were used in the morphological differentiation from dermatofibroma. In addition, in accordance with the literature, they found CD34 positive and factor XIIIa negative staining as opposed to dermatofibroma. One of the patients had dermatofibroma diagnosed before DFSP. Another case was diagnosed with dermatofibroma elsewhere in the body at the same time as DFSP diagnosis.

Initial treatment is surgical removal of the lesion. The purpose of the surgery is to remove the tumor by obtaining a negative surgical margin. Re-excision is recommended if surgical margin is positive.[17-19] One of the patient was diagnosed with punch biopsy before surgery. In our cases, surgical margin negativity could be obtained by re-excision in three cases. Surgical reexcision was not possible in a case and patient underwent radiotherapy.

Radiotherapy is also used as a primary therapeutic modality, as well as adjuvant therapy after surgery. A 10 -year disease-free survival rate after surgery and adjuvant radiotherapy was $93 \%$ in a single-center retrospective study. Postoperative radiotherapy is usually preferred if there is no positive surgical margin and no re-excision is possible. Adjuvant radiotherapy is not required if a negative surgical margin is obtained.[11,20] In our cases, because of surgical margin positivity, one of the patient needed radiotherapy. Other patients did not require adjuvant radiotherapy.

DFSP is characterized by translocation between chromosomes 17 and 22 [ $\mathrm{t}(17 ; 22)$ (q22; q13)]. This translocation results in PDGFRB overexpression. Imatinib mesylate, an inhibitor of protein tyrosine kinase in DFSP treatment, has been approved for adults in unresectable, recurrent and/or metastatic DFSP. If this translocation is absent in the tumor, it is unresponsive to imatinib treatment. [8] Since our case was not unresectable, recurrent or metastatic, this translocation was not looked at and this treatment was not applied.

\section{Conclusion}

DFSP skin and subcutaneous tissue-derived is skin sarcoma. As tumor with a local growth tendency and rarely metastasis, a large surgical excision with negative surgical margin is primer treatment. Close follow-up is required, especially for local recurrences. If no negative surgical margin is achieved, radiotherapy is recommended. Imatinib treatment is effective in patients with no local control and/or metastatic disease. 


\section{Disclosures Statement}

Ethics Committee Approval: This study was conducted inaccordance with local ethical rules.

Peer-review: Externally peer-reviewed.

Conflict of Interest: None declared.

Authorship contributions: Concept - Ü. Ü; Design - Ü. Ü, S. E. D; Supervision - Ü. Ü, N. K; Materials - Ü. Ü, S. E. D, F. Y; Data collection \&/or processing - Ü. Ü, N. K; Analysis and/or interpretation - Ü. Ü; Literature search - Ü. Ü, T. T. D; Writing - Ü. Ü, T. T. D; Critical review - Ü. Ü, F. Y.

\section{References}

1. Eguzo K, Camazine B, Milner D. Giant dermatofibrosarcoma protuberans of the face and scalp: a case report. Int J Dermatol 2014;53(6):767-72.

2. Paramythiotis D, Stavrou G, Panagiotou D, Petrakis G, Michalopoulos A. Dermatofibrosarcoma protuberans: a case report and review of the literature. Hippokratia 2016;20(1):80-3.

3. Trofymenko O, Zeitouni NC. Association of patient demographic characteristics with dermatofibrosarcoma protuberans tumour size at diagnosis in the U.S. National Cancer Database. Br J Dermatol 2017;177(4):e103-4.

4. Wales C, Caravaglio JV, Radi M, Woo R, Bancroft L. A rare case of dermatofibrosarcoma protuberans of the forefoot. Radiol Case Rep 2016;11(2):102-5.

5. Kreicher KL, Kurlander DE, Gittleman HR, Barnholtz-Sloan JS, Bordeaux JS. Incidence and Survival of Primary Dermatofibrosarcoma Protuberans in the United States. Dermatol Surg 2016;42 Suppl 1:S24-31.

6. Stamatakos M, Fyllos A, Siafogianni A, Ntzeros K, Tasiopoulou G, Rozis M, et al. Dermatofibrosarcoma protuberans: a rare entity and review of the literature. J BUON 2014;19(1):34-41.

7. Bogucki B, Neuhaus I, Hurst EA. Dermatofibrosarcoma protuberans: a review of the literature. Dermatol Surg 2012;38(4):537-51.

8. Rutkowski P, Van Glabbeke M, Rankin CJ, Ruka W, Rubin BP, Debiec-Rychter M, et al. Imatinib mesylate in advanced dermatofibrosarcoma protuberans: pooled analysis of two phase II clinical trials. J Clin Oncol 2010;28(10):1772-9.

9. Tsai YJ, Lin PY, Chew KY, Chiang YC. Dermatofibrosarcoma protuberans in children and adolescents: Clinical presentation, histology, treatment, and review of the literature. J Plast Reconstr Aesthet Surg 2014;67(9):1222-9.
10. Loghdey MS, Varma S, Rajpara SM, Al-Rawi H, Perks G, Perkins W. Mohs micrographic surgery for dermatofibrosarcoma protuberans (DFSP): a single-centre series of 76 patients treated by frozen-section Mohs micrographic surgery with a review of the literature. J Plast Reconstr Aesthet Surg 2014;67(10):1315-21.

11. Chen YT, Tu WT, Lee WR, Huang YC. The efficacy of adjuvant radiotherapy in dermatofibrosarcoma protuberans: a systemic review and meta-analysis. J Eur Acad Dermatol Venereol 2016;30(7):1107-14.

12. Stacchiotti S, Pantaleo MA, Negri T, Astolfi A4 Tazzari M, Dagrada GP, et al. Efficacy and Biological Activity of Imatinib in Metastatic Dermatofibrosarcoma Protuberans (DFSP). Clin Cancer Res 2016;22(4):837-46.

13. Wang C, Luo Z, Chen J, Zheng B, Zhang R, Chen Y, et al. Target therapy of unresectable or metastatic dermatofibrosarcoma protuberans with imatinib mesylate: an analysis on 22 Chinese patients. Medicine (Baltimore) 2015;94(17):e773.

14. Rutkowski P, Klimczak A, Ługowska I, Jagielska B, Wągrodzki M, Dębiec-Rychter $M$, et al. Long-term results of treatment of advanced dermatofibrosarcoma protuberans (DFSP) with imatinib mesylate - The impact of fibrosarcomatous transformation. Eur J Surg Oncol 2017;43(6):1134-41.

15. Sellheyer K, Nelson P, Krahl D. Dermatofibrosarcoma protuberans: a tumour of nestin-positive cutaneous mesenchymal stem cells? Br J Dermatol 2009;161(6):1317-22.

16. Yan X, Takahara M, Xie L, Tu Y, Furue M. Cathepsin K expression: a useful marker for the differential diagnosis of dermatofibroma and dermatofibrosarcoma protuberans. Histopathology 2010;57(3):486-8.

17. Kimmel Z, Alam M. Peripheral excision margins for dermatofibrosarcoma protuberans. Ann Surg Oncol 2008;15(9):2617.

18. Harati K, Lange K, Goertz O, Lahmer A, Kapalschinski N, Stricker I, et al. A single-institutional review of 68 patients with dermatofibrosarcoma protuberans: wide re-excision after inadequate previous surgery results in a high rate of local control. World J Surg Oncol 2017;15(1):5.

19. Flohil SC, van Lee CB, Beisenherz J, Mureau MAM, Overbeek LIH, Nijsten T, et al. Mohs micrographic surgery of rare cutaneous tumours. J Eur Acad Dermatol Venereol 2017;31(8):1285-8.

20. Castle KO, Guadagnolo BA, Tsai CJ, Feig BW, Zagars GK. Dermatofibrosarcoma protuberans: long-term outcomes of 53 patients treated with conservative surgery and radiation therapy. Int J Radiat Oncol Biol Phys 2013;86(3):585-90. 